\title{
Base Composition, Size and Sequence Similarities of Genome Deoxyribonucleic Acids from Clinical Isolates of Pseudomonas putrefaciens
}

\author{
By R. J. OWEN, RITA M. LEGROS AND S. P. LAPAGE \\ National Collection of Type Cultures, \\ Central Public Health Laboratory, \\ London $\mathrm{NW}_{9}{ }_{5} \mathrm{HT}$
}

(Received I8 August 1977)

\begin{abstract}
The mean base compositions of DNA from 27 strains of Pseudomonas putrefaciens, $P$. rubescens and $P$. piscicida ranged from 43.4 to $53.2 \mathrm{~mol} \% \mathrm{GC}$ with genome sizes from $3.04 \times 10^{9}$ to $4.23 \times 10^{9}$ daltons. On the basis of in vitro DNA-DNA binding, estimated spectrophotometrically from initial renaturation rates, $P$. putrefaciens strains were heterogeneous in the extent to which they shared similar nucleotide sequences, and were divided into four DNA homology groups. The DNA characteristics of strains in these groups correlated with several biochemical characteristics that facilitated identification of clinical isolates of $P$. putrefaciens. The two species $P$. putrefaciens and $P$. rubescens appear to be synonymous and none of the four groups of $P$. putrefaciens was related in DNA sequences to $P$. piscicida. Pseudomonas putrefaciens should therefore be retained as a single species and characteristics for identifying the various groups within the species are listed.
\end{abstract}

\section{INTRODUCTION}

The Gram-negative, rod-shaped bacterium Pseudomonas putrefaciens (Derby \& Hammer 193I) Long \& Hammer I94I was listed by Doudoroff \& Palleroni (1974) as a species incertae sedis in the eighth edition of Bergey's Manual of Determinative Bacteriology because it did not conform to the generic description of Pseudomonas. This species was excluded from Pseudomonas mainly because strains had DNA base compositions in the range 43.5 to $59.0 \mathrm{~mol} \% \mathrm{GC}$ (Colwell, Citarella \& Ryman, I965; Herbert et al., I97I ; Levin, I972; Lee, Gibson \& Shewan, I977), whereas the GC contents of typical Pseudomonas species range from 58 to $70 \%$ (Mandel, 1966).

Pseudomonas putrefaciens is widely distributed (Hugh, 1970); some strains have caused spoilage of fish and poultry (Shewan, I97I; Barnes \& Melton, 1971), while others have been isolated from human clinical specimens (Minagawa, I963; King, I964; von Graevenitz \& Simon, I970; Gilardi, I972; Riley, Tatum \& Weaver, I972; Fourquet et al., 1975). Some clinically isolated strains were aetiologically linked to disease in man although the resulting infections were relatively benign (Debois et al., I975; Holmes, Lapage \& Malnick, 1975; Rosenthal, Zuger \& Apollo, 1975).

Two groups of strains within $P$. putrefaciens were reported (Riley et al., r972; Levin, 1972; Holmes et al., I975): one with GC contents ranging from $47^{\circ} 8$ to $50.8 \%$ and unable to grow in $6 \% \mathrm{NaCl}$, the other with GC contents ranging from 55.9 to $59.0 \%$ and salt tolerant. Bacteriocins (Williams \& Levin, 1975) and autolysis (Levin \& Van Sickle, 1976) were also used to separate these groups.

Pseudomonas rubescens (Pivnick, 1955) and P. piscicida (Hansen, Weeks \& Colwell, 1965 ) resemble $P$. putrefaciens in having low GC contents of 44 to $48 \%$ (Mandel, Weeks \& Colwell, 1965; De Ley et al., 1966; Levin, 1972) and for this reason were also listed by 
Table I. Source of strains

\begin{tabular}{|c|c|c|}
\hline Species & Strain no. & Source \\
\hline Pseudomonas putrefaciens & 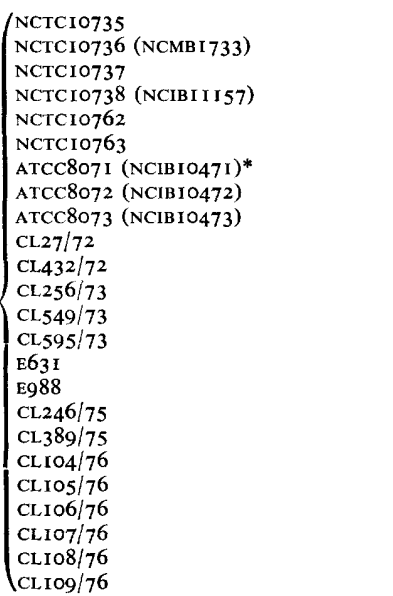 & 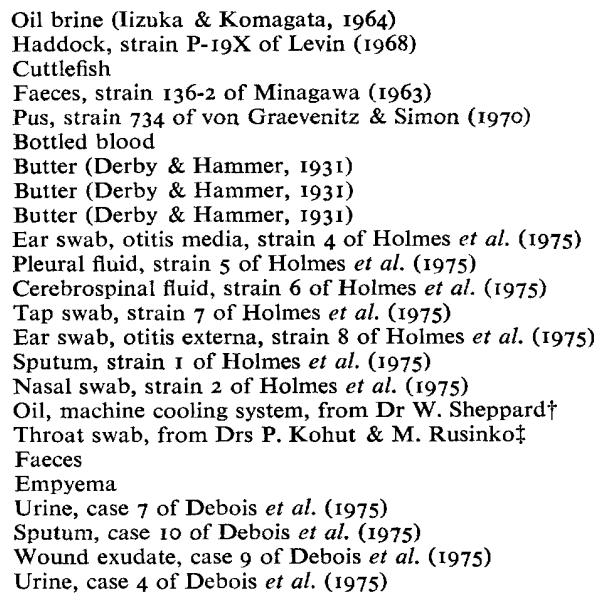 \\
\hline $\begin{array}{l}\text { Pseudomonas rubescens } \\
\text { Pseudomonas rubescens } \\
\text { Pseudomonas piscicida }\end{array}$ & $\begin{array}{l}\text { NCTCI0695 }(\mathrm{NClB} 8768, \text { ATCCI } 2099)^{*} \\
\text { ATCCI } 9857 \\
\text { ATCC1525I* }\end{array}$ & $\begin{array}{l}\text { Cutting oil (Pivnick, 1955) } \\
\text { Not known } \\
\text { Marine unspecified, strain I } 5 \text { of Hansen et al. (1965) }\end{array}$ \\
\hline
\end{tabular}

Doudoroff \& Palleroni (1974) as species incertae sedis. Pseudomonas rubescens and $P$. putrefaciens were reported to be similar to each other phenotypically (Hugh, 1970; Holmes et al., I975) and Lee et al. (1977), on the basis of a numerical taxonomic analysis, proposed that they be renamed Alteromonas putrefaciens. Members of the genus Alteromonas have base compositions of 43 to $48 \% \mathrm{GC}$ and are separated from species of Pseudomonas solely on this characteristic (Baumann et al., 1972).

This study investigated the taxonomic relationships based on DNA characteristics of strains classified as $P$. putrefaciens. The base compositions, genome sizes and base sequence similarities of DNA from various clinical isolates of $P$. putrefaciens are described and compared with those of $P$. putrefaciens strains from other sources and of type strains of $P$. rubescens and $P$. piscicida. Correlations between phenotypic and DNA characteristics are examined for tests that might improve the identification of potentially pathogenic clinical isolates of $P$. putrefaciens.

\section{METHODS}

Strains used and conditions for growth. Twenty-seven strains of Pseudomonas were used (Table I). Escherichia coli (strain B) NCTC10537 and Serratia marcescens NCTC1377 were used as DNA reference strains for the determination of base compositions and genome molecular weights. Bacteria were grown overnight at $30{ }^{\circ} \mathrm{C}$ in $200 \mathrm{ml}$ Nutrient Broth no. 2 (Oxoid) with aeration in an orbital incubator (Gallenkamp), except for $P$. piscicida ATCCI525I which was grown at $30{ }^{\circ} \mathrm{C}$ for $48 \mathrm{~h}$ in the above medium supplemented with $2.5 \%(\mathrm{w} / \mathrm{v}) \mathrm{NaCl}$.

DNA extraction and purification. Bacteria were harvested, washed and resuspended in $0.15 \mathrm{M}-\mathrm{NaCl}$ and 0. I M-EDTA buffer pH $8.0\left(5 \mathrm{~g}\right.$ wet wt in $50 \mathrm{ml}$ buffer) and lysed at $60^{\circ} \mathrm{C}$ with sodium dodecyl sulphate at a final concentration of $2 \%(\mathrm{w} / \mathrm{v})$. DNA was extracted and purified by a combination of the methods of Marmur (1961) and Brenner et al. (1969), which included treatment twice with $50 \mu \mathrm{g}$ ribonuclease $\mathrm{ml}^{-1}$ (bovine pancreas; BDH), treatment with $50 \mu \mathrm{g}$ pronase $\mathrm{ml}^{-1}$ (Koch-Light) and deproteinization with aqueous phenol. The DNA samples were precipitated with $95 \%(\mathrm{v} / \mathrm{v})$ ethanol and finally with either propan-2-ol or 2-ethoxyethanol. Samples were redissolved in 0.I $\times$ SSC buffer (0.I5 $\mathrm{M}-\mathrm{NaCl}$ buffered with $0.015 \mathrm{M}$-trisodium citrate at $\mathrm{pH} 7.0$ ) and stored at $4{ }^{\circ} \mathrm{C}$ over chloroform. Samples stored for a month or more were reprecipitated before use. DNA purity was assessed from the 280:260:230 nm extinction ratios (Marmur, 1961). 
Estimation of base composition. The mean base composition of the DNA was estimated from the thermal denaturation temperature $\left(T_{m}\right)$ by the method of Marmur \& Doty (1962). Thermal denaturation was carried out in a Pye Unicam SPI 800 spectrophotometer equipped with an SP 876 series 2 temperature programme controller and heated cell block; $\Delta E_{260}$ was recorded automatically. The temperature of the DNA solution in the cuvette was measured with a thermistor thermometer as described by Hill (1968). The DNA mean base composition, expressed as percentage guanine and cytosine to total base content (mol \% GC), was calculated from the $T_{m}$ determined in $0.1 \times$ SSC buffer, and expressed relative to the chemically determined value of 50.9\% for Escherichia coli strain в (De Ley, 1970) by the equation:

$$
\% \mathrm{GC}=50 \cdot 9+2.08\left(T_{m X}-T_{m E}\right)
$$

where $T_{m X}$ is the melting temperature of the unknown DNA in $0 \cdot 1 \times \mathrm{SSC}$ and $T_{m E}$ is the melting temperature of DNA from E. coli strain B in $0 \cdot 1 \times \mathrm{SSC}$ buffer.

Fragmentation of double-stranded DNA. The DNA concentration was adjusted to approximately $70 \mu \mathrm{g} \mathrm{ml}^{-1}$ in $5 \mathrm{ml} \mathrm{O} \cdot \mathrm{I} \times \mathrm{SSC}$ buffer. Solutions were sonicated for three periods of $\mathrm{I}$ min at $4{ }^{\circ} \mathrm{C}$ at a frequency of 20 to $25 \mathrm{kHz}$ and an amplitude of 2 to $5 \mu \mathrm{m}$ in a $100 \mathrm{~W}$ ultrasonic disintegrator (MSE). This yielded fragments of average molecular weight $2 \times 10^{5}$ to $3 \times 10^{5}$ (Owen \& Snell, 1976).

Measurement of DNA reassociation. Reassociation was determined spectrophotometrically. Changes in $E_{260}$ due to the reassociation of the denatured DNA fragments were measured on a recorder with a full scale deflection of $0 \cdot$ Io extinction units. The buffer solutions were filtered through a nitrocellulose membrane ( $0.2 \mu \mathrm{m}$ pore size; Sartorius Membranfilter GmbH, Göttingen, West Germany), and the final DNA concentration was adjusted to $60 \mu \mathrm{g} \mathrm{ml}^{-1}$. The sheared DNA in $\mathrm{I} \cdot 8 \mathrm{ml}$ o. I $\times$ SSC buffer was denatured at $95^{\circ} \mathrm{C}$ for $10 \mathrm{~min}$, and the $\mathrm{Na}^{+}$concentration was adjusted to $0.5 \mathrm{I} \mathrm{M}$ with $0.2 \mathrm{ml}$ of $5 \mathrm{M}-\mathrm{NaCl}$. To measure the binding between DNA from different strains, an equimolar mixture of the two DNAs in $0 \cdot 1 \times$ SSC was also prepared. The DNA solutions were then quickly transferred to preheated cuvettes in the spectrophotometer cell compartment at the optimum temperature of renaturation (TOR). This temperature was approximately $25^{\circ} \mathrm{C}$ below the DNA melting temperature in $0.5 \mathrm{I}$ M-sodium buffer, and was calculated from the equation: $\mathrm{TOR}=5 \mathrm{I} \cdot 9+(0 \cdot 47 \times \% \mathrm{GC})$.

The renaturation of the DNA fragments was monitored by the decrease in extinction during the initial $40 \mathrm{~min}$ of the reaction. The apparent renaturation rate constant $\left(k^{\prime}\right)$ expressed in arbitrary units was calculated from the equation of Gillis, De Ley \& De Cleene (I970): $k^{\prime}=v / C^{2}$, where $v$ is the initial renaturation rate expressed as the decrease in extinction at $260 \mathrm{~nm} \mathrm{~min}^{-1}$, and $C$ is the DNA concentration expressed in mM nucleotide pairs. The DNA concentrations were based on estimates determined chemically.

Estimation of genome size. The genome size (molecular weight) of a prokaryotic organism is the amount of double-stranded chromosomal DNA it contains expressed in numbers of base pairs or more commonly as its molecular weight. The renaturation rate constant of a DNA containing non-repetitive sequences was shown to be inversely proportional to its molecular complexity (Britten \& Kohne, 1966). Genome sizes were therefore calculated relative to a reference DNA (Escherichia coli strain B) with a mean molecular weight of $2 \cdot 7 \mathrm{I} \times 10^{9}$ (Gillis \& De Ley, 1975) from the formula:

$$
\text { Genome size }=\frac{k^{\prime}(E \cdot \text { coli }) \times 2 \cdot 71 \times 10^{9}}{k^{\prime}(\text { unknown })} .
$$

Estimation of DNA binding. The experiments to determine the degree of binding between DNA from different strains were done in $0.5 \mathrm{I}$ M-sodium buffer and at the TOR as described above. If the two DNAs in the mixture differed in base composition by $5 \% \mathrm{GC}$ or less, the mean of their respective TOR values was used. When GC differences exceeded $5 \%$, the TOR of the reference DNA was used. All determinations were repeated in duplicate or triplicate. The degree of binding in a mixture of two DNAs at approximately the same molar nucleotide pair concentration was calculated using the equation given by De Ley, Cattoir \& Reynaerts (1970):

$$
D=100\left[4 V_{\mathrm{M}}-\left(V_{\mathrm{A}}+V_{\mathrm{B}}\right)\right] / 2 V_{\mathrm{A}}
$$

where $V_{\mathrm{A}}, V_{\mathrm{B}}$ and $V_{\mathrm{M}}$ are the renaturation rates (change in extinction at $260 \mathrm{~nm} \mathrm{~min}^{-1}$ ) of DNA samples $(\mathrm{A}, \mathrm{B})$ and the mixture $(\mathrm{M})$ respectively.

\section{RESULTS}

\section{Mean DNA base compositions}

The base compositions of the 27 strains examined (Table 2) were broadly distributed in the range 43.4 to $53.2 \%$ GC within which were three narrower ranges. The 43.4 to $44.4 \%$ GC range comprised six strains: $P$. piscicida ATCCI525I, P. rubescens ATCCI9857 and 
Table 2. DNA base compositions of bacterial strains

\begin{tabular}{|c|c|c|c|c|}
\hline DNA source & Strain & $\begin{array}{c}\text { Mean } T_{m} \pm \text { S.D. } \\
\text { in o.I } \times \text { SSC } \\
\text { buffer* }\end{array}$ & $\underset{\mathrm{GC}}{\mathrm{Mean}}$ & Published values (mol \% GC) \\
\hline P. piscicida & ATCCI 525I & $71 \cdot 7 \pm 0 \cdot 10$ & $43 \cdot 4$ & 44.5 (Mandel et al., I965) \\
\hline P. rubescens & NCTC10695 & $71 \cdot 8 \pm 0.05$ & $43 \cdot 6$ & 46.9 (Lee et al., 1977) \\
\hline P. putrefaciens & ATCC 8071 & $71 \cdot 9 \pm 0.00$ & $43 \cdot 8$ & $\begin{array}{l}43.4 \text { and } 45.4 \text { (Colwell et al., I965), } 48 \cdot 4 \\
\text { (Levin, I972), 44.7 (Lee et al., I977) }\end{array}$ \\
\hline$P$. putrefaciens & CL246/75 & $72 \cdot 0 \pm 0 \cdot 10$ & $44 \cdot 0$ & \\
\hline$P$. rubescens & ATCCI9857 & $72 \cdot I \pm 0 \cdot 00$ & $44 \cdot 2$ & 46. I (De Ley et al., 1966), 48. I (Levin, 1972) \\
\hline \multirow{21}{*}{$P$. putrefaciens } & NCTCI0736 & $72 \cdot 2 \pm 0 \cdot 15$ & $44 \cdot 4$ & 45.9 (Lee et al., 1977), 49.3 (Levin, 1972) \\
\hline & ATCC 8073 & $73 \cdot 0 \pm 0.00$ & $46 \cdot I$ & $50 \cdot 1$ (Levin, 1972$)$ \\
\hline & CLIO6/76 & $73 \cdot 0 \pm 0.00$ & $46 \cdot I$ & \\
\hline & NCTCIO 0735 & $73 \cdot 1 \pm 0.05$ & $46 \cdot 3$ & \\
\hline & E63I & $73 \cdot I \pm 0.05$ & $46 \cdot 3$ & \\
\hline & CL549/73 & $73 \cdot 1 \pm 0.05$ & $46 \cdot 3$ & \\
\hline & $\mathrm{CLIO} / 76$ & $73 \cdot I \pm 0 \cdot 00$ & $46 \cdot 3$ & \\
\hline & NCTC 10737 & $73 \cdot 2 \pm 0.05$ & $46 \cdot 5$ & \\
\hline & CL389/75 & $73 \cdot 2 \pm 0 \cdot 10$ & $46 \cdot 5$ & \\
\hline & E988 & $73 \cdot 2 \pm 0 \cdot 10$ & $46 \cdot 5$ & \\
\hline & ATCC 8072 & $73 \cdot 5 \pm 0.00$ & $47 \cdot 2$ & $47 \cdot 3$ (Lee et al., 1977), 50. I (Levin, 1972) \\
\hline & CL256/73 & $74 \cdot 1 \pm 0 \cdot 10$ & $48 \cdot 4$ & \\
\hline & CL432/72 & $74 \cdot 2 \pm 0 \cdot 00$ & $48 \cdot 6$ & \\
\hline & NCTCIO 0738 & $75 \cdot 8 \pm 0 \cdot 10$ & $51 \cdot 9$ & $54 \cdot 7$ (Lee et al., 1977) \\
\hline & CLIO5/76 & $75 \cdot 8 \pm 0 \cdot 00$ & $5 x \cdot 9$ & \\
\hline & NCTCI 0762 & $76 \cdot 1 \pm 0.05$ & $52 \cdot 6$ & \\
\hline & CL27/72 & $76 \cdot 1 \pm 0.05$ & $\begin{array}{l}52 \cdot 6 \\
52 \cdot 6\end{array}$ & \\
\hline & $\begin{array}{l}\text { CL595/73 } \\
\text { CLIO8/76 }\end{array}$ & $\begin{array}{l}76 \cdot 1 \pm 0.30 \\
76 \cdot 2 \pm 0 \cdot 00\end{array}$ & $\begin{array}{l}52 \cdot 0 \\
52 \cdot 8\end{array}$ & \\
\hline & NCTC 10763 & $76 \cdot 2 \pm 0.05$ & $52 \cdot 8$ & \\
\hline & CLIO9/76 & $76 \cdot 3 \pm 0 \cdot 10$ & $53 \cdot 0$ & \\
\hline & $I_{\mathrm{CLIO}} 4 / 76$ & $76 \cdot 4 \pm 0.00$ & $53 \cdot 2$ & \\
\hline E. coli & NCTCI 0537 & $75 \cdot 3 \pm 0.05$ & $50 \cdot 9$ & \\
\hline
\end{tabular}

NCTC10695, and three strains of $P$. putrefaciens including the type strain ATCC807I. The $46 \cdot \mathrm{I}$ to $48 \cdot 6 \%$ GC range comprised I $2 P$. putrefaciens strains of which $\mathrm{Io}$ had base compositions of $46 \cdot \mathrm{I}$ to $47 \cdot 2 \% \mathrm{GC}$ and two (CL256/73 and CL432/72) had slightly higher base compositions of 48.4 and $48.6 \%$ GC. The $5 \mathrm{I} \cdot 9$ to $53.2 \% \mathrm{GC}$ range comprised the remaining nine strains of $P$. putrefaciens.

Our base composition results (Table 2 ) agreed with the previously published data on $P$. putrefaciens also given in Table 2 (Colwell et al., I965; Mandel et al., 1965; De Ley et al., I966; Lee et al., I977) but showed a consistent discrepancy of about $4 \%$ GC from the values of Levin (1972). This difference was attributed to our results being expressed relative to a value of $50.9 \% \mathrm{GC}$ (De Ley, 1970) for the DNA of $E$. coli strain B, whereas Levin (I972) reported a higher value of $54.0 \%$ for DNA of this strain.

\section{DNA renaturation rate constants}

The apparent renaturation rate constant $\left(k^{\prime}\right)$ values of DNA from the strains of $P$. putrefaciens, $P$. rubescens and $P$. piscicida were determined in $0.5 \mathrm{I}$ M-sodium buffer. Four selected DNAs (E. coli NCTCI0537, $S$. marcescens NCTCI 377, $P$. putrefaciens NCTCI0735 and $P$. rubescens NCTCI0695) were also tested in $0.39 \mathrm{M}$-sodium buffer $(2 \times \mathrm{SSC})$. This buffer was not used for the other DNAs as the renaturation rates were too slow for accurate measurement of gradients. The DNA of $E$. coli under our experimental conditions had an 
Table 3. DNA renaturation rate constants and bacterial genome sizes

\begin{tabular}{|c|c|c|c|c|c|c|c|}
\hline DNA source & Strain & Sodium molarity & $\begin{array}{l}\text { Temp- } \\
\text { erature } \\
\quad \text { of } \\
\text { renatur- } \\
\text { ation* } \\
\text { (TOR) }\end{array}$ & $\begin{array}{l}\text { No. of } \\
\text { determin- } \\
\text { ations }\end{array}$ & $\begin{array}{l}\text { Apparent } \\
\text { renaturation } \\
\text { rate constant } \\
\left(\mathrm{I} \mathrm{O}^{2} \times k^{\prime} \pm \text { S.D. }\right)\end{array}$ & $\begin{array}{l}10^{-9} \times \\
\text { genome } \\
\text { size } \\
\text { (daltons) }\end{array}$ & $\begin{array}{l}\text { Size } \\
\text { relative } \\
\text { to } \\
\text { E. coli } \mathrm{B}\end{array}$ \\
\hline P. rubescens & NCTC10695 & $\left\{\begin{array}{l}0.5 \mathrm{I} \\
0.39(2 \times \mathrm{SSC})\end{array}\right.$ & $\begin{array}{l}72 \cdot 4 \\
69 \cdot 2\end{array}$ & $\begin{array}{r}22 \\
2\end{array}$ & $\begin{array}{l}19.72 \pm 3.00 \\
12.81 \pm 0.19\end{array}$ & 3.038 & $I \cdot 12$ \\
\hline P. putrefaciens & $\left\{\begin{array}{l}\text { NCTC10762 } \\
\text { CL246/75 } \\
\text { E63I } \\
\text { CL27/72 } \\
\text { CL256/73 }\end{array}\right.$ & $\begin{array}{l}0.5 \mathrm{I} \\
0.5 \mathrm{I} \\
0.5 \mathrm{I} \\
0.5 \mathrm{I} \\
0.5 \mathrm{I}\end{array}$ & $\begin{array}{l}76 \cdot 6 \\
72 \cdot 6 \\
73 \cdot 7 \\
76 \cdot 7 \\
74 \cdot 6\end{array}$ & $\begin{array}{r}3 \\
2 \\
5 \\
4 \\
30\end{array}$ & $\begin{array}{l}19.32 \pm 0.44 \\
19.14 \pm 0.63 \\
18.92 \pm 1.36 \\
18.01 \pm 0.59 \\
17.88 \pm 1.26\end{array}$ & $\begin{array}{l}3 \cdot 101 \\
3 \cdot 131 \\
3 \cdot 167 \\
3 \cdot 327 \\
3 \cdot 351\end{array}$ & $\begin{array}{l}I \cdot I 4 \\
I \cdot I 6 \\
I \cdot I 7 \\
I \cdot 23 \\
I \cdot 24\end{array}$ \\
\hline P. rubescens & ATCCI9857 & $0.5 \mathrm{I}$ & $72 \cdot 7$ & 2 & $17.64 \pm 0.5 I$ & $3 \cdot 397$ & $I \cdot 25$ \\
\hline$P$. putrefaciens & $\left\{\begin{array}{l}\text { NCTC10738 } \\
\text { ATCC } 8071 \\
\text { CL432/72 } \\
\text { NCTC10763 } \\
\text { CL389/75 } \\
\text { CL595/73 } \\
\text { NCTCIO735 } \\
\text { CL549/73 } \\
\text { E988 } \\
\text { NCTCIO } 0737 \\
\text { CLI08/76 } \\
\text { NCTCIO } 736 \\
\text { CLIO6/76 } \\
\text { ATCC } 8072 \\
\text { ATCC } 8073\end{array}\right.$ & $\begin{array}{l}0.5 \mathrm{I} \\
0.5 \mathrm{I} \\
0.5 \mathrm{I} \\
0.5 \mathrm{I} \\
0.5 \mathrm{I} \\
0.5 \mathrm{I} \\
0.5 \mathrm{I} \\
0.39(2 \times \mathrm{SSC}) \\
0.5 \mathrm{I} \\
0.5 \mathrm{I} \\
0.5 \mathrm{I} \\
0.5 \mathrm{I} \\
0.5 \mathrm{I} \\
0.5 \mathrm{I} \\
0.5 \mathrm{I} \\
0.5 \mathrm{I}\end{array}$ & $\begin{array}{l}76 \cdot 3 \\
72 \cdot 5 \\
74 \cdot 7 \\
76 \cdot 7 \\
73 \cdot 8 \\
76 \cdot 6 \\
73 \cdot 7 \\
70 \cdot 6 \\
73 \cdot 7 \\
73 \cdot 8 \\
73 \cdot 8 \\
76 \cdot 7 \\
72 \cdot 8 \\
73 \cdot 6 \\
74 \cdot 1 \\
73 \cdot 6\end{array}$ & $\begin{array}{r}\text { I } 4 \\
4 \\
4 \\
4 \\
4 \\
4 \\
27 \\
2 \\
4 \\
5 \\
7 \\
2 \\
5 \\
4 \\
4 \\
5\end{array}$ & $\begin{array}{r}17 \cdot 47 \pm I .53 \\
17 \cdot 38 \pm I \cdot 18 \\
17 \cdot 31 \pm I \cdot 20 \\
17 \cdot 14 \pm 0.78 \\
17.07 \pm 0.72 \\
17.04 \pm 2 \cdot 15 \\
17.03 \pm I \cdot 38 \\
9.85 \pm 0.44 \\
16.81 \pm 0.89 \\
16.59 \pm 0.92 \\
16.01 \pm I \cdot 84 \\
15.92 \pm I \cdot 22 \\
15.86 \pm 0.99 \\
14.42 \pm I .43 \\
14.20 \pm 1.19 \\
14.19 \pm 0.89\end{array}$ & $\begin{array}{l}3 \cdot 430 \\
3 \cdot 448 \\
3 \cdot 462 \\
3 \cdot 496 \\
3 \cdot 510 \\
3 \cdot 516 \\
3 \cdot 523 \\
\\
3 \cdot 564 \\
3 \cdot 612 \\
3 \cdot 743 \\
3 \cdot 764 \\
3 \cdot 778 \\
4 \cdot 155 \\
4 \cdot 220 \\
4 \cdot 223\end{array}$ & $\begin{array}{l}\mathrm{I} \cdot 27 \\
\mathrm{I} \cdot 27 \\
\mathrm{I} \cdot 28 \\
\mathrm{I} \cdot 29 \\
\mathrm{I} \cdot 30 \\
\mathrm{I} \cdot 30 \\
\mathrm{I} \cdot 3 \mathrm{I} \\
\\
\mathrm{I} \cdot 32 \\
\mathrm{I} \cdot 33 \\
\mathrm{I} \cdot 38 \\
\mathrm{I} \cdot 39 \\
\mathrm{I} \cdot 39 \\
\mathrm{I} \cdot 53 \\
\mathrm{I} \cdot 56 \\
\mathrm{I} \cdot 56\end{array}$ \\
\hline$P$. piscicida & $\operatorname{ATCCI} 525 \mathrm{I}$ & $0.5 \mathrm{I}$ & $72 \cdot 3$ & 6 & $15 \cdot 22 \pm I \cdot 80$ & 3.937 & $\mathrm{I} \cdot 45$ \\
\hline E. coli & NCTCI0537 & $\left\{\begin{array}{l}0.51 \\
0.39(2 \times \mathrm{SSC})\end{array}\right.$ & $\begin{array}{l}75 \cdot 8 \\
73 \cdot 0\end{array}$ & $\begin{array}{l}4 \\
2\end{array}$ & $\begin{array}{l}22 \cdot I I \pm 0.95 \\
14.71 \pm 0.97\end{array}$ & $2 \cdot 710 \dagger$ & $1 \cdot 00$ \\
\hline S. marcescens & NCTCI 377 & $\left\{\begin{array}{l}0.5 \mathrm{I} \\
0.39(2 \times \mathrm{SSC})\end{array}\right.$ & $\begin{array}{l}80 \cdot 6 \\
78 \cdot 2\end{array}$ & $\begin{array}{l}4 \\
2\end{array}$ & $\begin{array}{l}15.10 \pm 1.01 \\
10.42 \pm 0.42\end{array}$ & 3.968 & $I \cdot 46$ \\
\hline
\end{tabular}

apparent rate constant of $22 \cdot \mathrm{II} \times \mathrm{IO}^{-2}$, which was similar to the two previously determined values for this DNA of $19 \cdot 1 \times 10^{-2}$ (Gillis et al., 1970) and $22 \cdot 2 \times 10^{-2}$ (Swings \& De Ley, 1975). The latter rates were determined using slightly different experimental conditions (approximately $80 \mu \mathrm{g} \mathrm{DNA} \mathrm{ml}^{-1}$ in $2 \times \mathrm{SSC}$ buffer) from those used here.

\section{Genome sizes}

The molecular weights of the genome DNAs from strains of $P$. putrefaciens and $P$. rubescens ranged from $3.04 \times 10^{9}$ to $4.23 \times 10^{9}$ (Table 3) which represented an overall size difference of $36 \%$. Differences in size of $0.5 \times 10^{9}$ or less were within the limits of experimental reproducibility and were not considered to be taxonomically significant. The genome size of $P$. piscicida ATCCI $525 \mathrm{I}\left(3.94 \times 10^{9}\right.$ daltons) was also within the $P$. putrefaciens range. All strains had genome sizes larger than that of $E$. coli strain B, in some cases by as much as $56 \%$, but they were within the range of $\mathrm{I} \times 10^{9}$ to $7 \times 10^{9}$ reported for the molecular weights of various other bacterial species (Bak, Christiansen \& Stenderup, 1970; Gillis \& De Ley, 1975). The DNA of Serratia marcescens, a micro-organism taxonomically unrelated to $P$. putrefaciens, was included as a reference standard for the estimation of genome 
Table 4. Reassociation of DNA samples from $P$. putrefaciens, $P$. rubescens and $P$. piscicida, with DNA from reference strains $P$. rubescens NCTCI0695 and $P$. putrefaciens NCTC10735

The extent of binding between DNA from the test strains and DNA from the reference strains is expressed as a percentage relative to the reference DNA using the formula: $D=100\left[4 V_{M}-\left(V_{A}\right.\right.$ $\left.\left.+V_{\mathrm{B}}\right)\right] / 2 V_{\mathrm{A}}$ where $V_{\mathrm{M}}$ is the renaturation rate of the mixture, $V_{\mathrm{A}}$ is the renaturation rate of the reference DNA, and $V_{\mathrm{B}}$ is the renaturation rate of the test strain. $\triangle \mathrm{GC}$ is the difference in the mean $\% \mathrm{GC}$ contents of the DNAs in the reassociation mixture. Genome sizes are expressed relative to that of the respective reference DNA.

Reference DNA from strain:

DNA source

DNA homology group I

$P$. rubescens NCrC10695

$P$. putrefaciens CL246/75

P. rubescens ATCCI 9857

$P$. putrefaciens ATCC $807 \mathrm{I}$

DNA homology group II

$$
P . \text { putrefaciens }\left\{\begin{array}{l}
\text { NCTCIO } 735 \\
\text { NCTCIO737 } \\
\text { ATCC } 8072 \\
\text { NCTCIO736 }
\end{array}\right.
$$

DNA homology group III

$$
\text { P. putrefaciens }\left\{\begin{array}{l}
\mathrm{CL} 256 / 73 \\
\mathrm{CL} 432 / 72 \\
\mathrm{CL} 549 / 73 \\
\mathrm{E} 631 \\
\mathrm{E} 988 \\
\mathrm{ATCC} 8073 \\
\mathrm{CL} 389 / 75 \\
\mathrm{CLIO6} / 76
\end{array}\right.
$$

DNA homology $\mathrm{g}_{\mathrm{r}}$ oup IV

\begin{tabular}{|c|c|c|c|c|c|}
\hline \multicolumn{3}{|c|}{ NCTCI0695 } & \multicolumn{3}{|c|}{ NCTC10735 } \\
\hline $\begin{array}{c}\text { Relative } \\
\text { binding } \\
(\%)\end{array}$ & $\Delta \mathrm{GC}$ & $\begin{array}{l}\text { Relative } \\
\text { genome } \\
\text { size }\end{array}$ & $\begin{array}{c}\text { Relative } \\
\text { binding } \\
(\%)\end{array}$ & $\Delta \mathrm{GC}$ & $\begin{array}{c}\text { Relative } \\
\text { genome } \\
\text { size }\end{array}$ \\
\hline
\end{tabular}

P. putrefaciens $\left\{\begin{array}{lr}\text { NCTCIO738 } & 25 \\ \text { NCTCIO762 } & 24 \\ \text { CL595/73 } & 6 \\ \text { CL27/72 } & 25 \\ \text { NCTCIO763 } & 1 \\ \text { ATCCI } 525 \mathrm{I} & 20\end{array}\right.$

$\begin{array}{rrrrrr}100 & 0 & 1 \cdot 00 & 47 & 2 \cdot 7 & 0.86 \\ 100 & 0.4 & 1 \cdot 03 & & & \\ 100 & 0.6 & I \cdot 12 & 41 & 2 \cdot 1 & 0.98 \\ 83 & 0.2 & 1 \cdot 14 & 26 & 2 \cdot 5 & 0.98\end{array}$

$\begin{array}{rrrrrr}39 & 2 \cdot 7 & \mathbf{I} \cdot \mathrm{I} 6 & \mathrm{I} 00 & 0 & \mathrm{I} \cdot 00 \\ 30 & 2 \cdot 9 & \mathrm{I} \cdot 23 & 83 & 0 \cdot 2 & \mathrm{I} \cdot 06 \\ & & & 58 & 0.9 & \mathrm{I} \cdot 20 \\ 32 & 0.8 & \mathrm{I} \cdot 24 & 57 & \mathbf{I} \cdot 9 & \mathrm{I} \cdot 07\end{array}$

$4 \cdot 8 \quad \mathrm{I} \cdot \mathrm{IO}$

$\begin{array}{lrr}32 & 2 \cdot I & 0.95 \\ 31 & 2 \cdot 3 & 0 \cdot 98 \\ 23 & 0 & \mathrm{I} \cdot 0 \mathrm{I} \\ 26 & 0 & 0.90 \\ \mathrm{I} 8 & 0.2 & \mathrm{I} \cdot 03 \\ 38 & 0.2 & \mathrm{I} \cdot 20 \\ \mathrm{I} 4 & 0.2 & \mathrm{I} \cdot 00 \\ 3 \mathrm{I} & 0.2 & \mathrm{I} \cdot \mathrm{I} 8\end{array}$

$\begin{array}{rrrrrr}25 & 8 \cdot 3 & \mathrm{I} \cdot \mathrm{I} 3 & \mathrm{I} 3 & 5 \cdot 6 & 0.97 \\ 24 & 9 \cdot 0 & \mathrm{I} \cdot 02 & 9 & 6 \cdot 3 & 0.88 \\ 6 & 9 \cdot 0 & \mathrm{I} \cdot \mathrm{I} 6 & 24 & 6 \cdot 3 & \mathrm{I} \cdot 00 \\ 25 & 9 \cdot 0 & \mathrm{I} \cdot \mathrm{IO} & 18 & 6 \cdot 3 & 0.94 \\ 1 \mathrm{I} & 9 \cdot 2 & \mathrm{I} \cdot \mathrm{I} 5 & 27 & 6 \cdot 5 & 0.99 \\ 20 & 0 \cdot 2 & \mathrm{I} \cdot 30 & 19 & 2 \cdot 9 & 1 \cdot 12\end{array}$

size, and our molecular weight value of $3.97 \times 10^{9}$ agreed with the $3.99 \times 10^{9}$ value previously reported for this species by Gillis \& De Ley (1975). However, both of these values for $S$. marcescens were less than the estimate of $5.56 \times 10^{9}$ reported by Bak et al. (I970).

\section{Estimates of interstrain DNA-DNA binding}

Accuracy of binding estimates. From a total of Ioo interstrain reassociations (including repeat determinations), the overall reproducibility of the spectrophotometric method for the estimation of DNA binding with our experimental conditions was $\pm 6 \%$ (pooled standard deviation). This compared well with the overall standard deviation for this method of $\pm 4.7 \%$ reported by De Ley et al. (I970), and $\pm 4.1 \%$ reported by Gibbins \& Gregory (1972). Binding values of $<20 \%$ were considered approximate estimates because of possible errors in measurements involving slow rates of reassociation.

DNA homology group I. Two strains of P. rubescens (NCTCI0695 and ATCCI9857) and two strains of $P$. putrefaciens (CL246/75 and ATCC807I) represented a DNA homology group with high levels of binding $(>83 \%$ ) relative to $P$. rubescens NCTCI0695, the selected 
Table 5. Reassociation of DNA samples from P. putrefaciens, $P$. rubescens and P. piscicida, with DNA from reference strains $P$. putrefaciens CL256/73 and NCTC10738

See Table 4 legend for details.

DNA source

DNA homology group I

P. rubescens NCTCI0695

$P$. putrefaciens ATCC807I

DNA homology group II

$$
P . \text { putrefaciens }\left\{\begin{array}{l}
\text { NCTC } 10735 \\
\text { NCTC } 10737 \\
\text { ATCC } 8072 \\
\text { NCTCIO } 0736
\end{array}\right.
$$

DNA homology group III

$$
\text { P. putrefaciens }\left\{\begin{array}{l}
\text { CL256/73 } \\
\text { CL432/72 } \\
\text { CL549/73 } \\
\text { E63I } \\
\text { E988 } \\
\text { ATCC8073 } \\
\text { CL389/75 } \\
\text { CL 106/76 }
\end{array}\right.
$$

DNA homology group IV

$$
P . \text { putrefaciens }\left\{\begin{array}{l}
\text { NCTCIO738 } \\
\text { NCTCIO762 } \\
\text { CL595/73 } \\
\mathrm{CL} 27 / 72 \\
\mathrm{NCTCIO} 6763 \\
\mathrm{CLIO} 8 / 76 \\
\mathrm{CLIO4/76} \\
\mathrm{CLIO9} / 76
\end{array}\right.
$$

\begin{tabular}{|c|c|c|c|c|c|}
\hline \multicolumn{3}{|c|}{$\operatorname{CL} 256 / 73$} & \multicolumn{3}{|c|}{ NCTC10738 } \\
\hline $\begin{array}{c}\text { Relative } \\
\text { binding } \\
(\%)\end{array}$ & $\Delta \mathrm{GC}$ & $\begin{array}{l}\text { Relative } \\
\text { genome } \\
\text { size }\end{array}$ & $\begin{array}{c}\text { Relative } \\
\text { binding } \\
(\%)\end{array}$ & $\Delta \mathrm{GC}$ & $\begin{array}{c}\text { Relative } \\
\text { genome } \\
\text { size }\end{array}$ \\
\hline 34 & $4 \cdot 8$ & $0.9 I$ & 27 & $8 \cdot 3$ & 0.89 \\
\hline 32 & $4 \cdot 6$ & $1 \cdot 03$ & & & \\
\hline 28 & $2 \cdot I$ & $1 \cdot 05$ & I3 & $5 \cdot 6$ & $\mathrm{I} \cdot 03$ \\
\hline $3 \mathrm{I}$ & $1 \cdot 9$ & $1 \cdot I 2$ & & & \\
\hline $2 I$ & $I \cdot 2$ & $I \cdot 26$ & & & \\
\hline 26 & $4 \cdot 0$ & $1 \cdot I 3$ & & & \\
\hline 100 & 0 & I. $\cdot 00$ & 40 & 3.5 & 0.98 \\
\hline 72 & 0.2 & $\mathrm{I} \cdot 03$ & & & \\
\hline $6 I$ & $2 \cdot I$ & $1 \cdot 06$ & & & \\
\hline 60 & $2 \cdot I$ & 0.95 & & & \\
\hline 48 & $I \cdot 9$ & I. 08 & & & \\
\hline 42 & $2 \cdot 3$ & $I \cdot 26$ & & & \\
\hline 45 & $\mathrm{I} \cdot 9$ & I.05 & & & \\
\hline $4 \mathrm{I}$ & $2 \cdot 3$ & $I \cdot 24$ & & & \\
\hline \multirow[t]{8}{*}{44} & 3.5 & $\mathrm{I} \cdot \mathrm{O} 2$ & 100 & 0 & $I \cdot 00$ \\
\hline & & & 96 & 0.7 & 0.90 \\
\hline & & & 97 & 0.7 & $1 \cdot 03$ \\
\hline & & & 87 & $0 \cdot 7$ & 0.97 \\
\hline & & & 87 & 0.9 & $I \cdot 02$ \\
\hline & & & 82 & 0.9 & $\mathrm{I} \cdot \mathrm{IO}$ \\
\hline & & & 90 & $I \cdot 3$ & 0.88 \\
\hline & & & 100 & $I \cdot I$ & 0.88 \\
\hline
\end{tabular}

P. piscicida ATCCI 525I
Reference DNA from strain:

DNA reference strain (Table 4). This DNA had lower levels of binding, ranging from 6 to $39 \%$, with DNA from Io other strains of $P$. putrefaciens. The four strains in this DNA homology group also had similar base compositions (mean $43.9 \pm 0.3 \% \mathrm{GC}$ ) and genome sizes (mean $3.25 \pm 0.20 \times 10^{9}$ daltons).

DNA homology group II. Table 4 also lists the degrees of binding between reference DNA from $P$. putrefaciens NCTCI0735 and DNA from 20 other strains. Three $P$. putrefaciens strains (NCTCI0735, NCTCI0737 and ATCC8072) formed a DNA homology group with binding values of 57 to $83 \%$ relative to NCTCI0735. The strains had similar base compositions (mean $46 \cdot 7 \pm 0.5 \% \mathrm{GC}$ ) and genome sizes except for the DNA of ATCC 8072 which was approximately $20 \%$ larger than the reference DNA. Pseudomonas putrefaciens NCTCI0736 was $57 \%$ related to NCTCI0735 and was included in this homology group, although its GC composition of $44.4 \%$ was lower than that of the other strains. There was $4 \mathrm{I}$ to $47 \%$ binding between NCTCI0735 and the two $P$. rubescens strains (NCTCI0695 and ATCCI9857) of group I, but the degrees of binding between NCTCI0735 and the remaining I $4 P$. putrefaciens strains were relatively low $(<38 \%)$.

DNA homology group III. Reference DNA from $P$. putrefaciens CL256/73 had degrees of binding ranging from $4 \mathrm{I}$ to $72 \%$ with eight $P$. putrefaciens strains (Table 5). Seven of these strains had GC compositions in the range $46 \cdot \mathrm{I}$ to $48.6 \%$ (mean $46 \cdot 9 \pm \mathrm{I} \cdot 0 \%$ ) and 
Table 6. Summary of DNA binding within and between the four P. putrefaciens DNA homology groups, and $P$. piscicida

\begin{tabular}{|c|c|c|c|c|c|}
\hline \multirow[b]{3}{*}{ DNA source } & \multicolumn{5}{|c|}{ Relative binding ( $\%$ ) with DNA from: } \\
\hline & \multicolumn{4}{|c|}{$P$. putrefaciens DNA group } & \multirow{2}{*}{$\begin{array}{l}P . \text { piscicida } \\
\text { ATCCI } 525 \mathrm{I}\end{array}$} \\
\hline & 1 & II & III & IV & \\
\hline $\begin{array}{l}P . \text { putrefaciens DNA gr } \\
\text { I } \\
\text { II } \\
\text { III } \\
\text { IV }\end{array}$ & $\begin{array}{l}83-100(4)^{*} \\
26-47(6) \\
29-34(4) \\
6-25(6)\end{array}$ & $\begin{array}{c}57-83(4) \\
14-38(11) \\
9-27(5)\end{array}$ & $\begin{array}{l}4 I-72(8) \\
40-44\end{array}$ & $82-100(8)$ & \\
\hline P. piscicida ATCCI $525 \mathrm{I}$ & 20 & 19 & 15 & NT & 100 \\
\hline
\end{tabular}

were included in the same homology group. They were more heterogeneous in degrees of DNA binding than members of the other homology groups. Pseudomonas putrefaciens CL256/73 and NCTC10738 (homology group IV, see below) were $44 \%$ related but binding with DNA from seven strains belonging to other homology groups was low $(<34 \%)$.

$D N A$ homology group $I V$. This group comprised eight strains (Table 5) with high levels of binding $(>82 \%$ ) relative to reference DNA from $P$. putrefaciens NCTCI0738. These strains and an additional strain (CLIO5/76) had similar GC compositions (mean 52.6 $\pm 0.4 \%$ ) and genome sizes (mean $3.38 \pm 0.24 \times 10^{9}$ daltons).

\section{Estimates of binding between $P$. putrefaciens homology groups and P. piscicida}

Table 6 summarizes the degrees of binding within each of the $P$. putrefaciens DNA homology groups, as well as the ranges of binding observed between DNA from strains in the different homology groups, and from P. piscicida ATCCI 525I. The highest degrees of intergroup binding were between strains of groups I and II $(47 \%)$, groups III and IV ( $44 \%$ ), and groups II and III (38\%). Pseudomonas piscicida ATCCI525I was not similar in nucleotide sequences to any of the $P$. putrefaciens reference strains, although it was similar in base composition to strains of DNA homology group I. Reassociations between NCTC 10738 ( $P$. putrefaciens DNA homology group IV) and $P$. piscicida were not carried out. The two strains had a difference of $8.5 \% \mathrm{GC}$ in their mean DNA base compositions and the maximum fraction of common GC regions shared by these two DNAs was estimated as only $26 \%$ (De Ley, I969). The taxonomic relationships of $P$. piscicida will not be discussed further here. For each DNA reassociation reaction the difference between the mean base compositions of the reassociated DNAs was calculated (see Tables 4 and 5). High levels of binding ( $72 \%$ and above) occurred only when the mean base compositions of the reassociated DNAs were similar $(\Delta \mathrm{GC}<\mathrm{r} \cdot 3 \%)$. The genome sizes of several of the reassociated DNAs differed and this was taken into account in the calculation of degrees of binding.

\section{DISCUSSION}

Strains of $P$. putrefaciens (Derby \& Hammer) Long \& Hammer I94I occur in a variety of clinical specimens and recently were associated with infections in man (Debois et al., I975; Holmes et al., I975; Rosenthal et al., 1975). The identification of this species by conventional bacteriological tests is not difficult, but strain variation in a number of biochemical characteristics has been recorded and it was suggested that the species might comprise several groups or possibly species (Levin, 1972; Riley et al., 1972; Holmes et al., 1975). Most of the strains studied here, which were isolated from a variety of 
Table 7. Characteristics for the practical differentiation of P. putrefaciens strains

\begin{tabular}{|c|c|c|c|c|}
\hline Characteristics & Group I & Group II & Group III & Group IV \\
\hline Total no. strains examined* & 4 & 4 & 9 & 9 \\
\hline No. strains of clinical origin & 0 & 0 & 7 & 9 \\
\hline Growth, $6 \% \mathrm{NaCl}$ & - & - & - & $+(1)$ \\
\hline Growth, $42{ }^{\circ} \mathrm{C}$ & - & - & $-(3)$ & $+(\mathrm{I})$ \\
\hline Growth, $37^{\circ} \mathrm{C}$ & + & - & + & + \\
\hline Growth, $5{ }^{\circ} \mathrm{C}$ & $+(1)$ & $+(\mathrm{I}) \S$ & $-(3)$ & - \\
\hline Acid from: Arabinose: & $+(\mathrm{I})$ & $+(\mathrm{I})$ & + & - \\
\hline Glucose & - & $+(\mathrm{I})$ & + & - \\
\hline Maltose & - & $+(\mathrm{I})$ & + & - \\
\hline Sucrose & - & $+(1)$ & $+(\mathrm{I})$ & - \\
\hline Growth, Salmonella/Shigella agar & $+(\mathrm{I})$ & - & $-(1)$ & + \\
\hline Alkali production, Christensen's citrate & - & $+(1)$ & $-(1)$ & - \\
\hline Mean GC \pm S.D. $(\mathrm{mol} \%)$ & $43 \cdot 9 \pm 0 \cdot 3$ & $46 \cdot I \pm I \cdot 2$ & $46 \cdot 9 \pm 1 \cdot 0$ & $52 \cdot 6 \pm 0.4$ \\
\hline
\end{tabular}

clinical and other sources, were not markedly different from each other in their overall genome sizes, yet the DNA base compositions and base sequence homologies showed that the species comprised genetically heterogeneous strains. As reported previously by Levin (1972), $P$. putrefaciens strains can have widely differing GC compositions and the range in values of 43 to $53 \%$ found here was large compared with observed ranges of most other bacterial species (Normore, I973).

Our DNA-DNA reassociation results provided evidence of at least four DNA homology groups within $P$. putrefaciens. Two of these groups (I and IV) had high levels ( $>82 \%$ DNA binding) of internal DNA relatedness whereas the other two groups (II and III) were characterized by comparatively greater heterogeneity in internal relatedness. It is difficult formally to relate DNA sequence relatedness and levels in the taxonomic hierarchy. Data on various members of the family Enterobacteriaceae (Brenner, 1973) indicated that strains classified in homogeneous phenetic species usually shared $70 \%$ or more of their DNA sequences. This may not necessarily be true for other groups of bacteria; species of Pseudomonas for example, show considerable heterogeneity in DNA relatedness (Palleroni et al., I972). However, on this criterion $P$. putrefaciens groups I and IV could constitute distinct species with only a small amount of DNA in common with each other. The division of strains into these DNA homology groups correlated well with the results on DNA base compositions and with several phenotypic characteristics obtained previously (Debois et al., 1975; Holmes et al., 1975).

To facilitate the practical identification of clinical isolates, the principal characteristics distinguishing the DNA groups of $P$. putrefaciens are summarized in Table 7. All clinical isolates of $P$. putrefaciens belonged either to group III or to group IV. However, there were no consistent differences in biochemical characteristics between the clinical isolates and those from marine and other sources, except that the clinical isolates were unable to grow at $5{ }^{\circ} \mathrm{C}$. Pseudomonas putrefaciens group IV was the most homogeneous group in DNA and phenotypic characteristics. Several characteristics $(6 \% \mathrm{NaCl}$ tolerance, growth on SS agar, lack of saccharolytic activity and GC contents of $53 \%$ ), suggested these strains were closely related to certain other clinical isolates of $P$. putrefaciens described as group 2 
(Riley et al., 1972), and the high GC content $(57.6 \%)$ strains of Levin (1972). None of these strains were proved to be human pathogens.

Doudoroff \& Palleroni (1974) considered that $P$. putrefaciens was not a valid species of Pseudomonas, and recently Lee et al. (1977) proposed that strains should be transferred, with the same specific epithet, to the genus Alteromonas (Baumann et al., 1972). The taxonomic problems regarding the generic status of these bacteria are outside the scope of the present study, but our results on P. putrefaciens DNA homology group I provided evidence of close similarities between the type strains of $P$. putrefaciens and $P$. rubescens (ATCC807I and NCTC10695 respectively). As concluded by Lee et al. (I977), these two species appear synonymous, and in accordance with the International Code of Nomenclature of Bacteria, 1976 Revision (Lapage et al., 1975) the name $P$. putrefaciens (Derby \& Hammer) Long \& Hammer I94I should be retained in preference to the junior synonym $P$. rubescens (Pivnick, 1955).

The estimation of degrees of DNA-DNA binding from the spectrophotometric measurement of renaturation rates has been used in relatively few taxonomic studies even though the method compares favourably in its accuracy and reproducibility with other reassociation techniques (Gibbins \& Gregory, 1972; Bradley, 1973; Martini \& Phaff, 1973; Crombach, 1974a; Swings \& De Ley, 1975). We found the method convenient because it did not need isotopically labelled DNA, any combination of cross-reactions between DNAs from different strains could be tested (reducing the likelihood of selecting an aberrant reference strain), and genome sizes could be estimated. In our view, the method was not suitable for use as a batch procedure or for estimating base mismatching from thermal denaturation of the duplexes (see also Crombach, 1974b).

It is concluded that the strains at present classified as $P$. putrefaciens should be retained in this species, but DNA relatedness studies should be extended to determine relationships between the various groups of $P$. putrefaciens and species of Alteromonas.

We thank Professor J. Vandepitte for cultures, B. Holmes for kindly providing the phenotypic results, and the Public Health Laboratory Service, which made this work possible.

\section{REFERENCES}

Bak, A. L., Christensen, C. \& Stenderup, A. (I970). Bacterial genome sizes determined by DNA renaturation studies. Journal of General Microbiology 64, 377-380.

Barnes, E. M. \& Melton, W. (I971). Extracellular enzymic activity of poultry spoilage bacteria. Journal of Applied Bacteriology 34, 599-609.

Baumann, L., Baumann, P., Mandel, M. \& Allen, R. D. (I972). Taxonomy of aerobic marine eubacteria. Journal of Bacteriology r1o, 402-429.

Bradley, S. G. (1973). Relationships among mycobacteria and nocardiae based upon deoxyribonucleic acid reassociation. Journal of Bacteriology $1 \mathrm{I3}, 645^{-6} \mathrm{I}$.

Brenner, D. J. (I973). Deoxyribonucleic acid reassociation in the taxonomy of enteric bacteria. International Journal of Systematic Bacteriology 23, 298-307.

Brenner, D. J., Fanning, G. R., Rake, A. V. \& Johnson, K. E. (1969). Batch procedure for thermal elution of DNA from hydroxyapatite. Analytical Biochemistry 28, 447-459.

Britten, R. J. \& Kohne D. E. (I966). Nucleotide sequence repetition in DNA. Carnegie Institution, Washington, Yearbook 65, 78-106.

Colwell, R. R., Citarella, R. V. \& Ryman, I. (1965). Deoxyribonucleic acid base composition and Adansonian analysis of heterotrophic, aerobic pseudomonads. Journal of Bacteriology 90, I I 48I 149.

Crombach, W. H. J. (I974a). Relationships among coryneform bacteria from soil, cheese and sea fish. Antonie van Leeuwenhoek 40, 347-359.

Crombach, W. H. J. $(1974 b)$. Thermal stability of homologous and heterologous bacterial DNA duplexes. Antonie van Leeuwenhoek 40, I33-144.

Debois, J., Degreef, H., Vandepitte, J. \& Spaepen, J. (1975). Pseudomonas putrefaciens as a cause of infection in humans. Journal of Clinical Pathology 28, $993-996$.

DE LEY, J. (I969). Compositional nucleotide distribution and the theoretical prediction of homology in bacterial DNA. Journal of Theoretical Biology 22, 89-1 16.

DE LEY, J. (I970). Reexamination of the association between melting point, buoyant density, and chemical base composition of deoxyribonucleic acid. Journal of Bacteriology 101, 738-754.

De Ley, J., Park, I. W., Tutgat, R. \& Van ErmenGEN, J. (I966). DNA homology and taxonomy of Pseudomonas and Xanthomonas. Journal of General Microbiology 42, 43-56.

De Ley, J., Cattoir, H. \& Reynaerts, A. (I970). The quantitative measurement of DNA hybridi- 
zation from renaturation rates. European Journal of Biochemistry 12, I33-142.

DeRBY, H. A. \& HAMmeR, B. W. (I93I). Bacteriology of butter. IV. Bacteriological studies on surface taint butter. Iowa Agricultural Experimental Station Research Bulletin 145, 389-4I6.

Doudoroff, M. \& Palleroni, N. J. (1974). Genus I. Pseudomonas Migula I 894. In Bergey's Manual of Determinative Bacteriology, 8th edn, p. 217. Edited by R. E. Buchanan and N. E. Gibbons. Baltimore: Williams \& Wilkins.

Fourquet, R., Coulanges, P., Goasguen, J. \& BoEhrer, J.-L. (1975). Premières souches de Pseudomonas putrefaciens isolées à Madagascar. Archives de l'Institut Pasteur de Madagascar 44, 49-55.

GILARDI, G. L. (1972). Infrequently encountered Pseudomonas species causing infections in man. Annals of Internal Medicine 77, 2 I I-2I 5.

Gibiins, A. M. \& GReGory, K. F. (I972). Relatedness among Rhizobium and Agrobacterium species determined by three methods of nucleic acid hybridization. Journal of Bacteriology III, I29I4I.

GILlIS, M. \& DE LEY, J. (1975). Determination of the molecular complexity of double-stranded phage genome DNA from initial renaturation rates. The effect of DNA base composition. Journal of Molecular Biology 98, 447-464.

Gillis, M., De Ley, J. \& De Cleene, M. (1970). The determination of molecular weight of bacterial genome DNA from renaturation rates. European Journal of Biochemistry 12, 143-1 53.

Hansen, A. J., Weeks, O. B. \& Colwell, R. R. (1965). Taxonomy of Pseudomonas piscicida (Bein) Buck, Meyers, and Leifson. Journal of Bacteriology 89, 752-76I.

Herbert, R. A., Hendrie, M. S., Gibson, D. M. \& SHEWAN, J. M. (197I). Bacteria active in the spoilage of certain sea foods. Journal of Applied Bacteriology 34, 4I-50.

HiLL, L. R. (I968). The determination of deoxyribonucleic acid base compositions and its application to bacterial taxonomy. In Identification Methods for Microbiologists, part B, pp. 177-186. Edited by B. M. Gibbs and D. A. Shapton. London: Academic Press.

Holmes, B., Lapage, S. P. \& Malnick, H. (1975). Strains of Pseudomonas putrefaciens from clinical material. Journal of Clinical Pathology 28, 149I 55 .

Hugh, R. (1970). A practical approach to the identification of certain nonfermentative Gramnegative rods encountered in clinical specimens. Public Health Laboratory 28, I68-I 87.

IIZUKA, H. \& Komagata, K. (I964). Microbiological studies on petroleum and natural gas. II. Determination of pseudomonads isolated from oilbrines and related materials. Journal of General and Applied Microbiology, Tokyo ro, 223-23I.

KING, E. O. (1964). The Identification of Unusual Pathogenic Gram-negative Bacteria, CDC Monograph. Atlanta, Georgia: Public Health Service, Department of Health, Education, Welfare.

Lapage, S. P., Sneath, P. H. A., Lessel, E. F., Skerman, V. B. D., Seeliger, H. P. R. \& Clark, W. A. (editors) (1975). International Code of
Nomenclature of Bacteria. Washington: American Society for Microbiology.

LeE, J. V., Gibson, D. M. \& Shewan, J. M. (I977). A numerical taxonomic study of some Pseudomonas-like marine bacteria. Journal of General Microbiology 98, 439-45I.

LEVIN, R. E. (I968). Detection and incidence of specific species of spoilage bacteria on fish. I Methodology. Applied Microbiology 16, I7341737 .

Levin, R. E. (1972). Correlation of DNA base composition and metabolism of Pseudomonas putrefaciens isolates from food, human clinical specimens, and other sources. Antonie van Leeuwenhoek 38, 12 I-I 27.

LeVIN, R. E. \& VAN SiCKLE, C. (1976). Autolysis of high-GC isolates of Pseudomonas putrefaciens. Antonie van Leeuwenhoek 42, I45-I 55 .

LONG, H. F. \& HAMmER, B. W. (I94I). Classification of organisms important in dairy products. III. Pseudomonas putrefaciens. Iowa Agricultural Experimental Station Research Bulletin 285, I 76-195.

Mandel, M. (1966). Deoxyribonucleic acid base composition in the genus Pseudomonas. Journal of General Microbiology 43, 273-292.

Mandel, M., Weeks, O. B. \& Colwell, R. R. (1965). Deoxyribonucleic acid base composition of Pseudomonas piscicida. Journal of Bacteriology. 90, I 492-I 493 .

MARMUR, J. (I961). A procedure for the isolation of deoxyribonucleic acid from micro-organisms. Journal of Molecular Biology 3, 208-218.

MARmur, J. \& Doty, P. (1962). Determination of the base composition of deoxyribonucleic acid from its thermal denaturation temperature. Journal of Molecular Biology 5, 109-1 18.

Martini, A. \& PhafF, H.J. (1973). The optical determination of DNA-DNA homologies in yeasts. Annali di Microbiologia ed Enzimologia 23, 59-68.

MinaGAwa, M. (I963). Studies on the strains closely related to Vibrio parahaemolyticus and reddish brown pigment-producing Pseudomonas isolated from the stools of patients of acute enteritis. Annual Report of the Institute of Food Microbiology, (Chiba University) 16, 9-23.

Normore, W. M. (I973). Guanine-plus-cytosine (GC) composition of the DNA of bacteria, fungi, algae and protozoa. In CRC Handbook of Microbiology, vol. II, Microbial Composition, pp. 585740. Edited by A. I. Laskin and H. A. Lechevalier. Cleveland: CRC Press.

OWen, R. J. \& SNell, J. J. S. (1976). Deoxyribonucleic acid reassociation in the classification of flavobacteria. Journal of General Microbiology 93, 89-IO2.

Palleroni, N. J., Ballard, R. W., Ralston, E. \& DoudorofF, M. (1972). Deoxyribonucleic acid homologies among some Pseudomonas species. Journal of Bacteriology mo, 1-1 I.

Pivnick, H. (1955). Pseudomonas rubescens, a new species from soluble oil emulsions. Journal of Bacteriology 7o, I-6.

Riley, P. S., TATum, H. W. \& Weaver, R. E. (1972). Pseudomonas putrefaciens isolates from clinical specimens. Applied Microbiology 24, 798-800.

Rosenthall, S. L., Zuger, J. H. \& Apollo, E. 
(1975). Respiratory colonization with Pseudomonas putrefaciens after near-drowning in salt water. American Journal of Clinical Pathology 64, 382-384.

Shewan, J. M. (197I). The microbiology of fish and fishery products - a progress report. Journal of Applied Bacteriology 34, 299-31 5 .

Swings, J. \& De Ley, J. (1975). Genome deoxyribonucleic acid of the genus Zymomonas Kluyver and van Niel 1936: base composition, size and similarities. International Journal of Systematic Bacteriology 25, 324-328.

von Graevenitz, A. \& Simon, G. (1970). Potentially pathogenic, nonfermentative, $\mathbf{H}_{2}$ S-producing Gram-negative rod (Ib). Applied Microbiology I9, 176.

Williams, J. L. \& Levin, R. E. (1975). Bacteriocin typing of Pseudomonas putrefaciens from food, human clinical specimens, and other sources. Antonie van Leeuwenhoek 4r, 97-100. 